\title{
SURVEILLANCE ROBOT AND TWO AXIS ARM MECHANISM FOR ARMY OPERATIONS
}

\author{
G.Nikitha, K.Varshita, M.Priyanka \\ Department of Information Technology, Stanley college of engineering and technology for women, \\ Hyderabad, Telangana, India \\ Prof Dr.A.Kanaka Durga \\ HOD, Department of Information Technology, Stanley college of engineering and technology for women, \\ Hyderabad, Telangana, India
}

\begin{abstract}
This paper presents a practical present-time approach for surveillance robots at remote locations and enemy territories using a remote controller based robotic vehicle on wireless technology that can be used for defense and military applications. This is used for detection of bombs, since detecting bombs manually contains high risk. So, in order to save their lives this is used. The camera is used to detect and identify human, objects etc. This vehicle is designed to work in limited area with better efficiency for example In Armed forces. They can use these types of robot vehicles in hostage situations to determine the number of terrorists in the building, types of weapons used, bombs etc. It also contains a two axis arm to hold objects. The processing unit used in proposed system is Arduino working on Embedded C. The Arduino controls the movement, gather information from camera that is used to stream the real time video of surrounding to the operator
\end{abstract}

Keywords - IOT, Robot, Surveillance, Arm mechanism.

\section{INTRODUCTION}

Robot is a machine which is designed to do a particular task. It is based on program i.e. software and hardware. Nowadays industry is becoming modern and they use automated technology to perform risky jobs. Surveillance is a real time collection and analysis of data that timely distributes the information to the operator. Surveillance in Defense Applications plays an important role for keeping an eye out in order to protect its citizens and take necessary action as needed. As many people are getting bordered, because of which it might be risky to observe all the things at that place. So, in order to reduce the risk a robot is used which monitors continuously. This robot continuously watch and send live streaming of it to the authorized person. Because of that monitoring work will be somewhat easy and it will be accurate because of technology. By the video surveillance user can keep an eye on the live field. The system operates remotely by using web server and entire data will be stored on cloud and only the authorized person can access the data whenever there is necessity.

Robots are usually small in size so they are enough capable to enter into tunnels, mines and small holes in building and also have capability to survive in harsh and difficult climatic conditions for life long time without causing any harm. This vehicle works within the range of the wifi. The system operates remotely by using web server and entire data will be stored on cloud and only the authorized person can access the data whenever there is necessity. This also contains a two axis arm in order to hold the objects and dissolve the bomb.

\section{LITERATURE SURVEY}

Till now from a detailed survey the robots are been controlled by using standard short distance RF waves which limits the capability, reduce the reliability and operational functionalities. The interfacing of sensors, camera module, Motor control and GPS is done with Raspberry pi for better processing control which provides better surveillance implementation in this system we obtain information about the area within $5 \mathrm{~m}-10 \mathrm{~m}$ by use of remote control, sensors and camera feed through wireless medium. This vehicle is designed to work in limited area with better efficiency for example in Armed forces. They can use these types of robot vehicles in hostage situations to determine the number of terrorists in the building, types of weapons used, bombs etc.

Most of the robots are used by charging the batteries as source of power. So, it becomes inconvenient to use this robot for long period. Existing robots use expensive video camera for live video streaming for manual control. This robotic vehicle with can widely be used as surveillance robot for security purpose and emergency rescue operations where human cannot enter.

The use of GSM modems ie, Global system for mobile communication is a time taking process since in this protocol 
instructions to the robot are sent through messages in fixed time slots and it waits until the reply of the instruction and then goes to the next instruction. With this there will be more network delay and the power consumption is also more.

\section{METHODOLOGY}

\section{PROPOSED SYSTEM}

Using internet communication, a real time mobile robot system is built by interfacing the microcontroller i.e. Arduino by using a serial port. The microcontroller is programmed in Arduino $\mathrm{C}$ language. The mobile robot system contain camera moving in two axis arm robot to hold objects or to dissolve the bomb.

\section{BLOCK DIAGRAM}

The below figure is the block diagram to show detailed connections between the components.

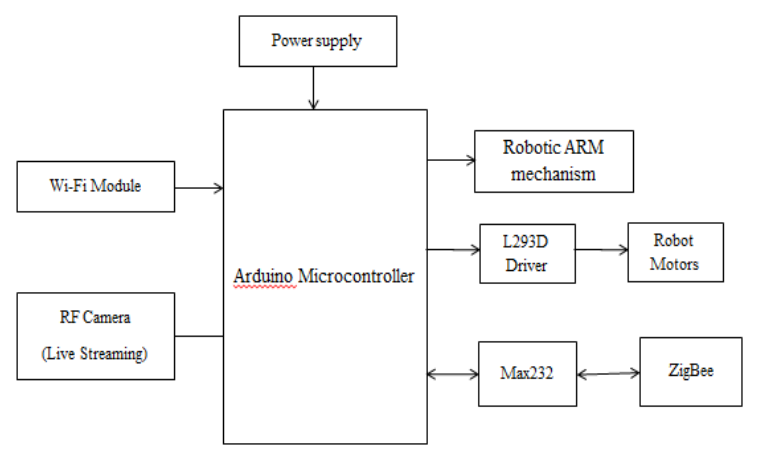

Figure:1 Block diagram

The power supply is given and then initialize the Arduino controller and load the HEX file then initialize the Wifi module by using IP address and port no. to establish network communication between hardware and PC. Zigbee module is connected with RS232 cables and the baud rate is selected as 9600.From webpage give commands for robotic direction control. From Zigbee serial window give commands for robotic arm movement control. The live streaming of data is observed on RF (radio frequency) live streaming page.

\section{RESULT}

This robot is used for surveillance and the communication is done through zigbee transmitter and receiver. This will replace the human soldier in hostage situations. The arm mechanism can be used to pick, place, dissolve the bomb based on the situation.

\section{FUTURE WORK}

The robot can be able to travel to a longer distance using a wireless range extender which helps to increase the signal strength.

Based on the current pandemic situation, i.e. COVID-19, this robot can also be used in order to monitor the patients health condition without the contact of human being.

\section{CONCLUSION}

In this we implement a smart surveillance robot for military application. With the help of this robot we know the real time condition of border area without using any human source. The surveillance robot gives us live streaming video according to that we give the command.

We can able to do video monitoring by using RF camera and also we are able to do live video surveillance for detecting harmful and dangerous objects like Bombs etc. By using zigbee $\mathrm{Tx}$ we can able to pick and place the harmful object and even diffuse the bomb if required.

\section{ACKNOWLEDGEMENT}

We take this opportunity to express our deep gratitude and appreciation to all those who encouraged us for successfully completing the major project work. We wish to express our sincere gratitude to Dr. SATYA PRASAD LANKA, Principal of Stanley College of Engineering and Technology for Women, Hyderabad for providing us with this opportunity. We thank, DR. A. KANAKA DURGA head of the department, Information technology, Stanley college of engineering and technology for women, for permitting us to undertake the major project work.

We would also like to express our gratitude to our guide, Dr. A.Kanaka Durga, HOD Department - IT , Stanley college of engineering and technology for women, under whose guidance and encouragement this project has been successfully completed.

We are also thankful to all the staff members of Information technology department of Stanley college of engineering and technology for women, for their invaluable support.

\section{REFERENCES}

[1] K.Damdhor , B.Vanitha \& K.Shanmugan. (2016). A Surveillance Robot For Real Time Monitoring And Capturing Controlled Using Arduino Mobile" , MiddleEast Journal of Scientific Research 24,pp 155-166.

[2] Vineela Kadiam, G. Pavani. (2014). Smart Phone Controlled Two Axes Robot for Video Surveillance Using Wireless Internet And Raspberry Pi Processor, 
International Journal of Research in Advent Technology, $2(10)$.

[3] Pavan.C, Dr. B. Sivakumar, (2012). Wi-Fi ROBOT FOR VIDEO MONITORING \& SURVEILLANCE SYSTEM , International Journal of Scientific \& Engineering Research Volume 3, Issue 8.

[4] Salunkhe Disha, Jagtap Ashwini, Ghodekar Darshana , Shelke Poonam. (2018). Wireless Multifunctional Robot for Military Applications, International Research Journal of Engineering and Technology, Volume 5 , Issue 3.

[5] Aniket.A.More , Akshay.V.Jadhav \& Sweta Patil. (2019). Surveillance Robot for Defence Environment , Volume 6 , Issue 2 .

[6] V. Vikram Gnanaraj, P.Ranjana, P. Thenmozhi. (2019). Patient Monitoring and Control System using Internet of Thing, International Journal of Innovative Technology and Exploring Engineering (IJITEE) ISSN: 2278-3075, Volume-8, Issue-6S3 .

[7] Tarunpreet Kaur, Dilip Kumar . (2015) . "Wireless Multifunctional Robot for Military Applications", IEEE 2 nd international conference on recent advances in engineering and computational sciences April 2017.

[8] Zadid Shifat, A. S. M., Md Saifur Rahman, Md FahimAlFattah, and Md ArifurRahman. (2014) . "A practical approach to microcontroller based smart phone operated robotic system at emergency rescue scheme" In Strategic Technology (IFOST), 9th International Forum on, pp. 414417. IEEE.

[9] Pavithra, S., and S. A. Siva San kari. (2013) . "7TH sense-a multipurpose robot for military" In Information C communication and Embedded Systems (ICICES), International Conference on, pp. 1224-1228. IEEE.

[10] M.Karthikeya and Mr. G. Sreeram . (2014) . "Intelligent Exploration and Surveillance Robot In Defense Environment" International Journal of Advanced Research in Electrical, Electronics and Instrumentation Engineering, Vol. 3,pp120-123.

[11] Balaji.M, Karthick.S, Manikandan.V, Benedict Ebinesar.J, Vijay Nagaraj. (2017). "Survillance and Target Engagement using Robots" IOSR Journal of Electronics and Communication Engineering, pp.01-06.

[12] M. Khamis, A. M. Elmogy, and F. O. Karray .(2011). "Complex Task Allocation in Mobile Surveillance Systems," Journal of Intelligent and Robotic Systems, vol. 64, no. 1, pp. 33-55. 